\title{
Effects of Dietary Soy and Its Constituents on Human Health: A Review
}

\author{
Fasiha Ahsan*1, Muhammad Imran ${ }^{1}$, Syed Amir Gilani ${ }^{1}$, Shahid Bashir ${ }^{1}$, Amar Ahmad Khan ${ }^{1}$, Anees Ahmed \\ Khalil $^{1}$, Faiz-ul Hassan Shah ${ }^{1}$ and Muhammad Hanif Mughal $^{2}$ \\ ${ }^{1}$ Faculty of Allied Health Sciences, Pakistan \\ ${ }^{2}$ Department of Biochemistry, Pakistan
}

Received: 眥: December 10, 2018; Published: 制: December 19, 2018

*Corresponding author: Fasiha Ahsan, Senior Lecturer and Consultant Dietician, Faculty of Allied Health Sciences, Pakistan

\begin{abstract}
Soy Protein occurs naturally in Soybean and is known to have many benefits to health. It is an alternate source of protein for those people who don't eat meat but along with benefits. This review has been undertaken to make people familiar with the importance of soy beans in clinical set up and in the food industries. Soybeans are legumes which include peanuts, chickpeas, other beans and pulses. Soybeans are highly versatile beans can be processed into oil, flour and meal. Approved health claims for soy protein in different countries is about $25 \mathrm{~g} /$ day which is known to reduce total cholesterol and LDL. Soy has different effective substance in it like Trypsin inhibitors, saponins, fibers, isoflavones and phytic acid. It has observed that soy shows some toxic effects. There are many studies raising concerns about the potential negative effects of it, due to the presence of allergens, also they contain anti nutrient agents who inhibit micronutrient absorption, it may also cause hypothyroidism, being deficient in a few essential amino acids, it is considered as an incomplete protein source. It may also cause hormonal consequences in infants in their later life.
\end{abstract}

Keywords: Soy Protein; Blood Pressure; Obesity; Drawbacks of Soy

\section{Introduction}

Soy was first grown in Asia but not to be used as a food. In early times of its manufacture, it was originally used only for crop production and was believed not suitable for human consumption, or not considered safe to use unless being fermented. Before 1920 's, soy was grown only as an animal feed or as a by-product of Soy Oil. But later people identified its methods of cooking and made it a part of their diet. After that for some years it is widely used in many countries like America, Hong Kong, Korea, South East Asia, Japan and China. Although India is searching for its possible uses as an alternate protein source for its economically backward, poor and vegetarian population. Soy Protein is abundantly found in a legume known as Soybean. The protein derived from soybeans and has many benefits to the health. They play a cardio protective role and possess estrogenic properties. Soy protein is often used as an alternative protein source in infant feeding formulas (Baby milk) particularly for those infants who are intolerant to cow milk protein and also a protein substitute for vegetarians [1].

Soybeans are primarily consumed by humans after being fermented and turned into a curd, tofu, a blend cheese-like substance made from the whey of fermented soybeans, is the most common example of this type of soybean application. It contains about $40 \%$ of protein, $35 \%$ carbohydrate, $20 \%$ fattyoil and $5 \%$ ash. It is one of the few plants that provide a complete protein and is therefore often used as a substitute of meat and dairy products because soy protein is quite stable at high temperatures, it is particularly a staple of many Chinese, Korean, Japanese and Southeast Asian dishes. Another common food use is in milk substitutes, such as soy milk. Soybeans are highly versatile, and the beans can be processed into oil, flour, and meal. Each of these has many dietary uses, and soy one of the accomplished dietary legumes. There are many properties about the health benefits of soybeans consumption; some scientist said that isoflavones in soybeans helps in the prevention of cancer and FDA recently certified that consumption of soybeans can help to lower the cholesterol level. However, the other study showed that raw soybean flour persuaded pancreatic cancer in laboratory rats, although these rats were fed excess amount of that which normal humans would normally ingest.

\section{Potential Role of Soy on Cardiovascular Disease}

Many substances related with soy protein have been involved in decreasing cholesterol including trypsin inhibitors, saponins, 
stanols sterols, fiber, isoflavones and phytic acid. Trypsin is a serine protease that is found in digestive system. Studies showed that in soybean high level of trypsin inhibitors i.e. 1-5\% of total protein is present. In vivo studies using rats, pancreatic cancer is observed when rat is exposes to high levels of trypsin inhibitor, while moderate levels cause the rat pancreas to more responsive to cancer causing agents. The US FDA infer that low levels of trypsin inhibitors present in soybeans cause no harm to human health. To destroy trypsin inhibitors, humans should consume soybeans cooked with "wet" heat. Phytic acid has a chelating effect that's why it is accused for reducing vital minerals especially when the person is already on low minerals and vitamins diet.

Phytic acid in soybean seeds binds to metals and minerals to form phytates i.e. chelated form of phytic acid with zinc, iron, magnesium and calcium, thus preventing the body to use these essential minerals especially zinc [2]. Much of the activity of trypsin inhibitors is ruined when soy products are treated with heat. A hypocholesterolemia effect by increasing the release of cholecystokinin from small amounts of heat-stable Bowman-Birk inhibitors may be applied. It then induces bile acid production from cholesterol and helps to exclude cholesterol through the gastrointestinal tract. However, animal examinations have not shown hypocholesterolemic effect when trypsin inhibitors were included in diet. Saponins are basically a group of triterpene and steroid glycosides. Two mechanisms are there that describe saponins effects on lowering cholesterol: Particularly saponins with structural features make some insoluble compounds with cholesterol like the precipitation cholesterol with digitonin.

Whenever this process occurs in gut, it inhibits the absorption and utilization of cholesterol by intestines. By forming mixed micelles saponins disturbs the hepatic circulation of bile acids. The reabsorption of bile acids from ileum is effectively blocked [3]. Stanols and sterols found soy oils decrease blood cholesterol by lowering the uptake of cholesterol from diet. These should be recommended for decreasing the LDL cholesterol to adults [4]. Epidemiological studies show a positive effect of whole soy foods and its products in reducing cardiovascular disease risk. In a Japanese study showed that higher intake of soybean (>101 g/ day) lowered the mortality rate of CVD [5]. Diverse soy foods differ in their biological effectiveness and defensive properties. Their estrogenic effects safeguard against severe rise in CVD threat after menopause, while internally originating estrogen level falls [6]. Consumption of more than 4 servings of broccoli, carrots and tofu or soy beans in a week decreased the danger of hypertension as compared to the consumption of less than 1 serving in a week [7].

Previous investigators studied in animals that the amino acids methionine and lysine kindle cholesterol level, in contrast arginine has a contrary effect. Soy proteins has more proportion of arginine than lysine and methionine as compared to animal protein sources. Two animal studies show that a mixture of L-amino acids similar to the model of soy protein had a cholesterol lowering effect that was not as formerly declared as that of hydrolyzed whole soy protein. Some other substances in soy protein might have a helpful effect greater than that of the protein alone. The greater ratio of arginine to lysine of soy protein may lower glucagon and insulin production, which leads to inhibition of synthesis of fats. Phytoestrogens can rapidly increase endothelial nitric oxide formation, enhance vasodilation and improve blood flow, providing anti-atherosclerotic effect. These effects on glucagon and insulin levels have been seen in hypercholesterolemic individuals. Isoflavones containing soy protein noticeably lower cholesterol as compared to soy proteins that don't contain isoflavones, in humans. Increase thyroxine levels with the intake of soy protein has been studied in animals.

Increase thyroxine level were hypothesized to decrease cholesterol levels, but studies on humans vary [8]. The hypothesis that soy protein decreases cholesterol by improved bile removal has been studied widely. Cholesterol excreted from the body as bile enables the liver to furnish more cholesterol for higher bile acid production and raises LDL receptors working. The conclusion is improved LDL elimination from the blood. Nevertheless, in human studies soy has not demonstrated to enhance bile acid elimination through feces [9].

\section{Soy Protein Effects on LDL Receptors}

Soy beans have 2 kinds of storage proteins such as globulin $11 \mathrm{~S}$ and globulin 7S. These globulins provoke working of LDL receptors. Clinical studies suggest that intake of soy protein increases the number of LDL receptors in humans. The mRNA levels in mononuclear cells of LDL receptors were much more in individuals who were fed with soy protein as compared to those fed with casein [10]. Fiber Investigators have stated that soy fiber decreases cholesterol level in hypercholesterolemic individuals. Other researches have recounted that soy fiber decreases cholesterol when it is in combination with other foods but when it is in combination with soy proteins it does not further decrease cholesterol [11]. Phytic acid, myoinositol hexaphosphate, exist in all soy proteins products that are not fermented and is very heatstable. Phytic acid binds with zinc readily in intestines, lowering its absorption. A deficiency of copper or high proportion of zinc than copper leads to increased blood cholesterol. The theory raised is that soy foods have both phytic acid and copper which may decrease cholesterol by lowering the zinc to copper ratio [12].

Isoflavones exist in all soy flours and in concentrated and isolated forms manufactured by a water removing process. These are plant-based estrogens and are biologically active in humans. The main source of isoflavones in foods is soy, which contain genistein, diadzein and glycetein. The advantageous effects of estrogen are decreased LDL cholesterol and increased HDL cholesterol. Phytoestrogens presumptuously have an identical but less effective impact. $20 \%$ of diet containing soy proteins have isoflavones restrains the formation of atherosclerotic lesions in simians. Geinstein is identified to hinder tyrosine kinase, an enzyme which works in the series of event which lead to formation of lesions and thrombi. The vascular effects of isoflavones are seen in humans. In one study, dietary isoflavones intake was associated with decrease aortic stiffness, particularly in older women. It contributes in reducing blood pressure. Isoflavones also have anti- 
oxidative properties and reduce oxidation of LDL. They enhance systemic arterial flexibility in women without effecting lipid levels of blood too. These studies demonstrate that isoflavones and other ethanol-soluble soy phytochemicals have direct impact on vascular system, regardless of the relationship with lipid metabolism [13].

\section{Hypotensive Effect of Soy}

High blood pressure raises the hazard of vascular damage by favoring inflammatory processes, thus increasing the risk of CVD. Moreover, level of Angiotensin II, a potential vessel constrictor, raises in high blood pressure and upsurge the action of lipoxygenase enzyme, which generates free radicals in the smooth muscles [14]. Soy and its constituents lower blood pressure by dilating vessels and hindering a significant enzyme implicated in controlling the blood pressure. Isoflavones lower blood pressure by focusing on mechanisms included in dilation of vessels, especially by interacting with the genetic response to estrogen, interrelated to nitric oxide (NO) synthase, in the interior lining of the vessels, raising nitric oxide production, which enhances blood flow through arteries [15]. Supplementing menopausal women, with Isoflavones, enhanced lumen dilation of vessels [16]. Constituents other than Isoflavones have revealed to own blood pressure lowering qualities. Blood pressure lowering effect of isoflavones by communicating with estrogen-response component of gene that raise the endothelial nitric oxide which boost up arterial blood flow [17]. The consequences of isoflavones on endothelial depends upon persons potential to convert daidzein into equol [18]. Soy nuts contain both soy protein and isoflavones diminished systolic blood pressure in healthy and metabolic syndrome persons and remarkable reduction in diastolic blood pressure in equol producing persons. Soy food contribution to satiety. Soy flour Muffins evoke greater feeling of fullness in hypercholesterolemic overweight adult [8].

Studies documented that soy protein snacks reduce appetite and also decreases the sugary snacks consumption among adults. High soy protein breakfast reduce serum appetite hormone ghrelin [19]. Pulp of Soy containing oligopeptides and high fiber content, has shown to defy angiotensin converting enzyme (ACE) action, specifying systematic proof of lowering blood pressure [20]. The US food and drug administration accepted the claims that the foods containing soy protein are beneficial against coronary heart diseases, and therefore their labeling as protective against coronary heart diseases was approved in October 1990.This decision was based on the studies according to which 25g of soy/ day reduces total cholesterol as well as low density lipoprotein cholesterol. This $25 \mathrm{~g}$ was divided into 4 servings/day, each serving containing 6.25g [12]. Effect of soy protein in cholesterol lowering was studied in humans and reported in 1967. According to the study if hypercholesterolemic men replace mixed protein with isolated soy protein at an intake of $100 \mathrm{~g} /$ day, it will lower mean cholesterol by less than 2.59mmol/L [21]. Between 1977 and 1994, 38 controlled clinical studies were published, their meta-analysis infers that placing animal protein with soy protein reduces total cholesterol, LDL cholesterol and triglycerides, but has no significant effect on HDL. Daily soy consumption along with diet low in saturated fat resulted $10 \%$ reduction in cholesterol, $12 \%$ in LDL while $11 \%$ in triglycerides. Systematic investigation deduced that replacing animal protein with soy protein lowers total cholesterol, LDL cholesterol and triglycerides in blood [22]. The nutrition committee of American heart association analyzed 22 random trials published since 1999, from among which 19 were carried out with hyperlipidemic subjects.it was gauged that mean consumption of $50 \mathrm{~g} /$ day (ranging from 25 to $133 \mathrm{~g}$ ) of isolated soy protein when compared with milk or other protein reduces LDL cholesterol by $3 \%$ but has no significant effect on HDL, TG, lipoprotein and blood pressure [13].

\section{Hypoglycemic Effect of Soy}

Diabetic patients have increase blood glucose concentration either due to the reduce production of insulin by $\beta$-cell of pancreas or due to the action of the insulin Hyperglycaemia increases the risk of coronary heart diseases and atherosclerotic lesion. Isoflavones effect on glycaemic control Other component of soy food (fiber, saponin, polyscharides) has also glucose lowering effect Isoflavones in soy protein decreases islet of Langerhans ?-cell loses and increases antioxidant activity [23]. Genistien as isoflavones genistien is an isoflavone, naturally occurring in many plants and it's has antioxidative activity it inhibits tyrosine kinases and improve insulin secretion by increasing the production of a-cells. Glucose stimulate insulin secretion and protect from program cell death through mechanism [24]. In postmenopausal women insulin sensitivity increases by consuming $54 \mathrm{mg}$ purified isoflavones genistien for one year and also reduce the chance of osteoporosis [5].

\section{Effect of Soy on Inflammation and Obesity}

Immune responses (Figure 1) utilize growth factors, prostaglandins, and cytokines. Healthy endothelial cell transfers into disease tissue. Soy isoflavones genistien is anti-inflammatory and antioxidative activity it suppresses the inflammatory gene expression and protect endothelial damage [25]. In obese postmenopausal women isoflavones extract rise the level of leptin, adiponectin and tumour necrosis factor alpha after supplementation for six months [26]. In soy protein Glycine and amino acid inhibit inflammatory pathways in rats and increases antioxidative activity [27]. In obesity (Figure 2) adipocytes secrete inflammatory cytokines and chemokine's that enhance pro-inflammatory state and also promote insulin resistance and low adiponectin levels [28]. Isoflavones reduce trunk fat cell, leptin concentration and serum lipid levels. Post-menopausal obese women take $75 \mathrm{mg}$ isoflavones for 1 year and reduce body fat mass [29]. Biologically mobile soy peptide has hypercholesterolemic effect through LDL-C receptors and bile acid ruling. $25 \mathrm{~g}$ soy lower risk of heart disease. Soy protein provide defense against Low density lipoprotein cholesterol, free radical damage, endothelial damage and also from kidney disturbance [30]. Soy proteins phytoestrogens are diphenolic similar to mammalian estrogen are Genistien, daidzien, glycitein and exert physiological influences $>1000$-folds as compare to endogenous estrogen [31]. 


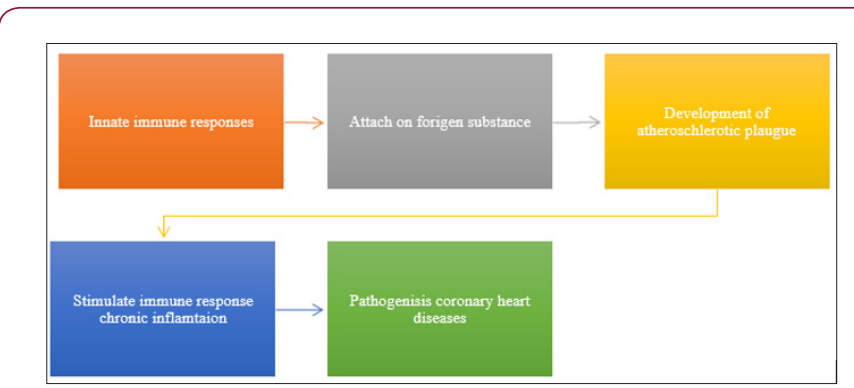

Figure 1: Soy protein and inflammation.

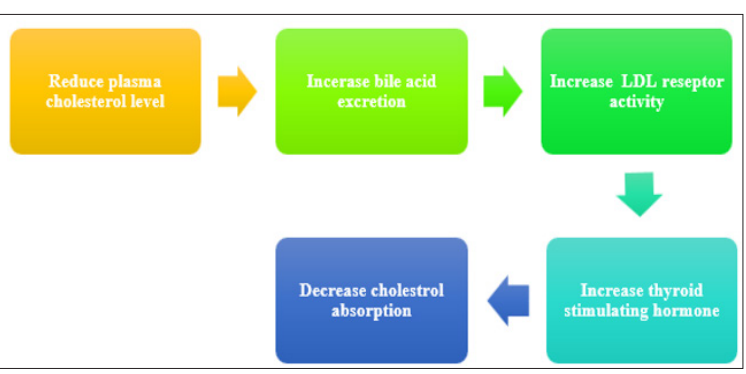

Figure 2: Role of soy in obesity reduction.

\section{Soy Protein Supplements}

According to a research $4.5 \mathrm{~g}$ /day Black Soy Peptide (BSP) supplementation was introduced in the form of tablets to 64 overweight/obese subjects for 12 weeks. After 12 weeks BSP introduced subjects showed significantly decreased body weights, body fat mass, body fat $\%$ and plasma leptin. It showed a great role in weight and fat mass regulation in overweight subjects, on the other hand it altered the leptin levels in their bodies [32]. In another research $100 \mathrm{~g}$ soy fiber/day was introduced in the form of Soy Fiber Supplemented Biscuits on daily basis for straight 12 weeks to 39 overweight/obese adults which showed significantly decreased levels of LDL-C, TC and BMI right after 12 weeks [33].

\section{Drawbacks of Soy Proteins on Human Health}

Along with a wide range of benefit there are also some disadvantages which are mostly overlooked by the consumer. According to Food Agriculture Organization, Soy protein is one of the 8 most common food Allergens. Usually, allergic reactions first occur in infants and children under the age of 3 , and many of children among them carry the allergy till they reach the age of 10 . Soy allergy can be cause by soy inhalation or ingestion. Symptoms caused by soy allergy are: stuffy or runny nose, rash, wheezing, itching in the mouth, nausea, vomiting or diarrhea, anaphylactic shock (Occurs rarely, can be a danger to life by affecting breathing and can only be treated by Epinephrine injections). Although $25 \mathrm{~g}$ of soy protein is recommended for cardiovascular disease patients to reduce the symptoms, but excess intake can also lead to allergic reactions [34]. Phytates are present in grain products and can bind structurally with iron, zinc, calcium, and other nutrients, thus blocking heir absorption in body. Soybean has the highest amount of phytate as compared to all other legumes, and the phytates of soybeans, are highly resistant to slow or long cooking, and soaking.
Among soybean products, Soy milk is particularly high in phytates, whereas tofu has moderate phytate content. Those countries where the use of tofu and soy milk is a part of culture are highly susceptible to developing deficiency related diseases the results of calcium, magnesium, iron, and zinc deficiency can become significant as it may also show symptoms of Rickets. Soybeans have some non-nutrient component such as Phytic acid, soya toxin, saponins, goitrogens, phytoestrogens and trypsin inhibitors which lower the mineral absorption. The method used to deactivate such anti nutrient components is through fermentation which is only applicable to a few (not all) soy products such as Soy and Tofu are usually used in unfermented form. The fermentation of some soy foods enables consumers to enjoy soy's nutritional benefits.

Thus individuals consuming a vegetarian diet typically including soy beans are more prone to such mineral deficiencies specially that of calcium, zinc and magnesium. The anti-nutrients subtances in modern soy products, such as those present in soy flour, can reduce protein digestion and cause intestinal problems and lead to chronic nutrient deficiencies by hindering their absorption. A deficiency of some essential nutrients and the interaction of Phytic acid with protein, vitamins, and minerals at the time of processing are the major reasons for the low nutritive value of soy isolate. Soy foods have a component (Goitrogens) which blocks the iodine absorption and thus exhibit anti thyroid properties. Isoflavone (A bioactive component) of Soybean contain such compounds inhibit the activity of Thyroid Peroxidase, an enzyme necessary for normal thyroid function [35]. A study of people with clinical features of hypothyroidism done in 2011 identified a progressive rate to obvious hypothyroidism. Such people should limit their soy intake or they might need to discuss with their doctor about modifying their thyroid medication [36].

Goiter and other Thyroid Diseases can also occur in children after the excessive consumption of Soy Isoflavones. The Genistein promote Apoptosis (programmed cell death) of Male testicular cell, thus it can affect male fertility [37]. It increases feminine characteristics in the body. Polycystic Ovarian Syndrome may also occur as a consequence of eating excess soy foods as they are usually Genetically Modified. A study done on a sample of males with higher soy consumption. Results showed that they have the low sperm counts as compared to those men who consume no soy foods [38]. Another survey found that the mean testosterone levels of adult subjects reduced by $19 \%$ after using soy protein powder four weeks [39]. Sometimes people face easy bleeding even with a mild hit, in such case vitamin K rich foods are introduced to promote the blood clotting but the case is different when consuming soybean. Soybean is an anti-coagulant (a substance that inhibits blood clotting). Despite consuming vitamin $\mathrm{K}$ rich foods (Green Leafy Vegetables) along with soybean, the anti-coagulant property of soybean does not change. Many people are low in vitamin K. Soy's anti-coagulant property is linked with its anti-trypsin activity.

Trypsin-an enzyme essential for the digestion of protein. Also, trypsin causes vitamin B12 to be absorbed. The soybean is 
an anti-trypsin agent thereby blocking Trypsin activity. So Soy increases the deficiency of vitamin K and B12 [40]. Soy milk is free of cholesterol and that is why it is suitable for consumption by heart disease patients but cholesterol being a vital substance for proper development of the brain and central nervous system in infant, can cause developmental problems. An enquiry done on soy formula fed infants showed amount of estrogenic compounds as much as 22,000 times more in soy milk-based formula than those in human breast milk. So, it created a question that such an increased consumption might result in abnormal development of organs at later stage of puberty in male infant [41]. The soy isoflavones (plant pigments) in soy (daidzein and genistein) are much known for their healthy advantages. What is not known to consumer is the research based fact that soybean consumption may lead to poor mineral absorption, infertility, low thyroid activity, and low energy levels. Poor cognitive test performance, Brain atrophy, and low brain weight cases are being reported in association with higher soy intake.

\section{Conclusion}

It is concluded that the use of soybeans $25 \mathrm{~g} /$ day shows advantageous change in cholesterol level and improve HDL, reduce LDL. As a phytoestrogen deals with vasodilation and having antioxidative properties. In premenopausal shows some refinement in arterials elasticity. Pakistan shows low production of soybeans globally which might be due to lack of knowledge regarding its different production aspects. On the other side, there may be a dark side to soy's vogue and fame. There are some doubts and health concerns related to the use of soy foods, many studies reflect that there can be a possible disruption of thyroid, hormonal and sexual function. A wide range of studies link soy to digestive distress, malnutrition, immune system breakdown, cognitive decline, infertility and even cancer. Soy foods are for sure a good to use but natural or fermented soy foods should be preferred rather than processed forms.

\section{References}

1. Dervries MC, Phillips S (2015) Potential role of soy protein on human health. J Food Sci 44: 121-133.

2. Sherif M (2013) Soybean, nutrition and health. J Nutr 20: 461-463.

3. David O (2014) Soy Proteins, saponins and plasma cholesterol. J Nutr 131: 29-71.

4. Martijin B, Katan M (2012) Efficacy and safety of plant sterols and stanols in management of blood cholesterol levels. Clin Proce 78: 965966.

5. Squadrito F, Marini H, Bitto A, Altavilla D, Polito F, et al. (2013) Genistein in the metabolic syndrome: Results of a randomized clinical trial. J Clin Endocrinol Metab 98(8): 3366-3374.

6. Zhan S, Ho S (2015) Meta-analysis of the effects of soy-protein containing isoflavones on the lipid profile. Am J Clin Nutr 81: 397-408.

7. Borgi L, Muraki I, Satija A, Willett W, Rimm E (2016) Soy protein consumption and the incidence of hypertension in three prospective cohort studies. J Hyper 67: 288-293.

8. Padhi EM, Ramdath D, Carson S, Hawke A, Blewett H, et al. (2015) Soy flour muffins over time and the impact of a health claim on willingness to consume. Food Res Int 77(3): 491-497.
9. Noriko H, Kenji S (2016) Evidence of existing a resistant protein that captures bile acid and stimulates fecal excretion. J Food Sci 1: 28442845.

10. Sirtori CW, Eberinil I, Arnold A (2015) Hypocholesteremic effects of soya proteins. Br J Nutr 97: 8-22.

11. Dan R, Emily M, Padhi S, Sarfraz S, Renwick A (2017) Cholesterol lowering effect of soy protein: a review of the effects of dietary soy and its constituents on risk factors for cardiovascular disease. Nutr 9(4): 3-24.

12. Erdman J (2013) AHA Science Advisory: Soy protein and cardiovascular disease: A statement for healthcare professionals from the Nutrition Committee of the AHA. Circul 102: 2555-2559.

13. Chao W (2014) Health Effects of soy protein and soy isoflavones in humans. J Nutr 138: 1244-1245.

14. Ross R (2012) Atherosclerosis an inflammatory disease. N Engl J Med 340: 115-126.

15. Colacurci N, Chiantera A, Fornaro F, Manzella D, Arciello A, et al (2012) Effects of soy isoflavones on endothelial function in healthy postmenopausal women. J Nutr 12: 299-307.

16. Jackson RL, Greiwe J, Schwen R (2015) Emerging evidence of the health benefits of S-equol, an estrogen receptor beta-agonist. Nutr Rev 69: 432448.

17. Hugel HM, Jackson N, May B, Zhang A, Xue C (2016) Polyphenol protection and treatment of hypertension. Phyto 23(2): 220-231.

18. Acharjee S, Zhou J, Elajami T, Welty F (2015) Effect of soy nuts and equol status on blood pressure, lipids and inflammation in postmenopausal women stratified by metabolic syndrome status. Metabol 64(2): 236243.

19. Konig D, Muser K, Berg A, Deibert P (2012) Fuel selection and appetite-regulating hormones after intake of a soy protein-based meal replacement. Nutr 28(1): 35-39.

20. Nishibori N, Kishibuchi R, Morita K (2017) Soy pulp extract inhibits angiotensin converting enzyme (ACE) activity in vitro: Evidence for its potential hypertension-improving action. J Diet Suppl 14(3): 241-251.

21. Anderson JW, Johnstone BM, Barnes S (2015) Meta-analysis of the effects of soy protein intake on serum lipids. N Engl J Med 333: 276-82.

22. Stradling C, Hamid K, Fisher S, Taheri G, Thomas F (2013) A Review of dietary influences on cardiovascular health: The role of dietary nutrients cardiovascular and hematological disorders drug targets. J Metabol 13(3): 208-230.

23. Younis N, Charlton V, Sharma R, Soran H, Durrington P (2014) Glycation of LDL in non-diabetic people: Small dense LDL is preferentially glycated both in vivo and in vitro. Athero 202: 162-168.

24. Gilbert ER, Liu D, Hue L (2013) Anti-diabetic functions of soy isoflavone genistein: Mechanisms underlying effects on pancreatic $\beta$-cell function. Food Fun 4(2): 200-212.

25. Libby P (2016) Inflammation and cardiovascular disease mechanisms. Am J Clin Nutr 83: 456S-460S.

26. Llaneza P, Gonzalez C, Fernandez J, Alonso A, Diaz F, et al. (2012) Soy isoflavones, diet and physical exercise modify serum cytokines in healthy obese postmenopausal women. Phyto 18: 245-250.

27. Mauriz JL, Matilla B, Culebras JM, Gonzalez P (2015) Dietary glycine inhibits activation of nuclear factor kappa $b$ and prevents liver injury in hemorrhagic shock in the rat. Radic Biol Med 31: 1236-1244.

28. Deboer, M (2013) Obesity, systemic inflammation and increased risk for cardiovascular disease and diabetes among adolescents: A need for screening tools to target interventions. Nutr 29(2): 379-386.

29. Wu J, Oka J, Tabata I, Higuchi M, Toda T, et al. (2016) Effects of isoflavone and exercise on BMD and fat mass in postmenopausal Japanese women: 
A 1-year randomized placebo-controlled trial. J Bone Miner Res 21: 780789.

30. McGraw N, Krul E, Grunz E, Parrish A (2016) Soy-based renoprotection. World J Nephrol 5(3): 233-257.

31. Barnes S, Prasain J, Alessandro T, Arabshahi A, Botting N, etal. (2011) The metabolism and analysis of isoflavones and other dietary polyphenols in foods and biological systems. Food Fun 2(5): 235-244.

32. Kwak JH, Ahn C, Park SH, Jung S, Min B, et al. (2015) Weight reduction effects of a black soy peptide supplement in overweight and obese subjects: double blind, randomized, controlled study. Food Func 3: 1019-1024.

33. Hu X, Gao J, Zhang Q, Fu Y, Li K, et al. (2013) Soy fiber improves weight loss and lipid profile in overweight and obese adults: A randomized controlled trial. Mol Nutr Food Res 57(12): 2147-2154.

34. Christopher T (2014) Soy protein allergy: Incidence and relative severity. J Nutr 134: 1213-1219.

35. Jenkins DJ, Kendall CW, D Costa MA, Jackson CJ, Vidgen E, et al. (2013) Soy consumption and phytoestrogens: effect on thyroid functioning of human body. J Metabol Nutr 169: 507-11.

ISSN: 2574-1241

DOI: 10.26717/BJSTR.2018.12.002239

Fasiha Ahsan. Biomed J Sci \& Tech Res (C) (7) This work is licensed under Creative

Submission Link: https://biomedres.us/submit-manuscript.php
36. Mercola G (2012) Soy protein role in nutritional deficences. J Nutr 120 : $1101-1105$

37. Kumar NB, Cantor A, Allen K, Riccardi D, Besterman Dahan K, et al. (2015) The specific role of isoflavones in reducing prostate cancer risk. Prostate. J Med 59: 141-7.

38. Dalais FS, Meliala A, Wattanapenpaiboon N, Frydenberg M, Suter DA, et al. (2014) Effects of a diet rich in phytoestrogens on prostate-specific antigen and sex hormones in men diagnosed with prostate cancer. J Urol 64: $510-5$

39. Hamilton Reeves JM, Rebello SA, Thomas W, Slaton JW, Kurzer MS (2016) Isoflavone-rich soy protein isolate suppresses androgen receptor expression without altering estrogen receptor-beta expression or serum hormonal profiles in men at high risk of prostate cancer. J Nutr 137: 1769-75.

40. Gallagher JC, Satpathy R, Rafferty K, Haynatzka V (2014) The effect of soy protein on nutrient metabolism. J Clin Nutr 11: 290-8.

41. Setchell KD, Zimmer Nechemias L, Cai J, Heubi JE (2013) Isoflavone content of infant formulas and the metabolic fate of these phytoestrogens in early life. Am J Clin Nutr 68: 1453S-1461S.

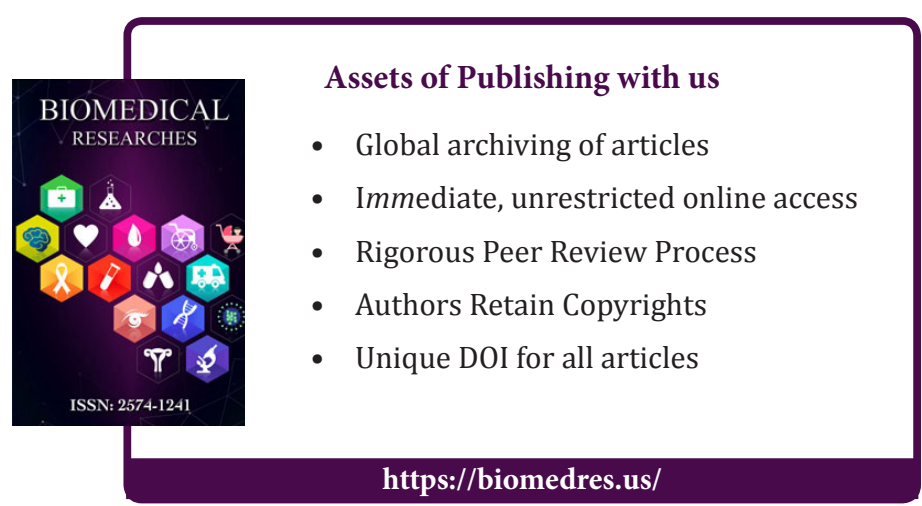

